\title{
Kernos
}

Revue internationale et pluridisciplinaire de religion grecque antique

16 | 2003

Varia

\section{A.-F. MORAND, Études sur les Hymnes orphiques}

\section{Carine Van Liefferinge}

\section{(2) OpenEdition}

\section{Journals}

Édition électronique

URL : http://journals.openedition.org/kernos/850

DOI : $10.4000 /$ kernos.850

ISSN : 2034-7871

Éditeur

Centre international d'étude de la religion grecque antique

Édition imprimée

Date de publication : 1 janvier 2003

Pagination : 379-380

ISSN : 0776-3824

Référence électronique

Carine Van Liefferinge, «A.-F. morand, Études sur les Hymnes orphiques », Kernos [En ligne], 16 | 2003, mis en ligne le 14 avril 2011, consulté le 22 septembre 2020. URL : http://journals.openedition.org/ kernos/850 ; DOI : https://doi.org/10.4000/kernos.850 
chute, l'apothéose. L'article est suivi par la liste des films ayant Orphée comme personnage principal ou secondaire.

L'ouvrage se termine sur trois traductions des vers 14 à 41 du livre $X$ : Pierre du Ryer (1660), l'abbé Banier (1732), Georges Lafaye (1926). On notera avec amusement que la traduction de Pierre du Ryer fait sept lignes de plus que ses successeurs. C'est ce qu'on appelle « une belle infidèle». Un ouvrage utile, qui constitue un bon moyen d'approcher une oeuvre à laquelle les travaux récents, comme celui de G. Tronchet, ont enfin rendu justice.

Alain Moreau

(Université de Montpellier)

Anne-France Morand, Études sur les Hymnes orphiques, Leiden, Brill, 2001. 1 vol. $16 \times 24,5 \mathrm{~cm}, \mathrm{xI}+374 \mathrm{p}$. (Religions in the Graeco-Roman World, 143). ISBN : 90-04$12030-0$.

Cette étude commence par la reproduction du texte grec des Hymnes orphiques dans l'édition de Quandt (Berlin, 1955) avec quelques leçons différentes adoptées par l'A. et justifiées en notes. Après une brève introduction qui présente ces quatre-vingt-sept hymnes et annonce le but ambitieux de l'ouvrage, à savoir une relecture de ces textes à la lumière de nouveaux documents, tels les feuilles d'or et le papyrus de Derveni, et, partant, une redéfinition de l'orphisme à partir de l'ensemble des documents mis sous le nom d'Orphée, s'ouvre le premier chapitre d'un ouvrage qui en compte cinq. Ce chapitre traite de la question du genre des hymnes et, adoptant la division en "invocation », « développement » et « demande » proposée par Rudhardt, en examine les différentes parties pour aborder ensuite une étude globale du corpus envisagé cette fois dans son ensemble comme un tout cohérent ordonné logiquement. Ainsi, si chaque hymne peut être envisagé isolément, l'ensemble du traité n'en atteste pas moins des conceptions religieuses cohérentes. Au terme de cette étude formelle, une brève conclusion reste plus descriptive que significative malgré l'affirmation de l'A. qui dit s'être démarquée des autres historiens de la religion grecque. Le deuxième chapitre aborde le contenu des Hymmes avec la question des offrandes contenues dans leurs titres. Cette question en entraîne d'autres, sur le rapport entre l'offrande et le dieu qui la reçoit, les autres rituels décrits dans les textes et la communauté qui les pratiquait. Les conclusions sur ces questions restent floues et suggèrent à l'A. l'existence d'une communauté religieuse réelle, encore imprécisée à cet endroit de l'ouvrage. Le chapitre III veut cerner les croyances relatives aux dieux contenues dans ces textes afin de déterminer si elles correspondent à celles d'un groupe orphique. Les mises en rapport avec les cuvres littéraires et les inscriptions témoignent d'un ancrage dans la tradition assorti d'innovations propres aux textes attribués à Orphée. Quant à la date et au lieu d'origine de ces Hymnes, là encore, malgré le peu d'éléments permettant de les préciser - les références à Éleusis et à Dionysos qui y sont contenues ne sont pas un indice suffisant -, l'A. propose, sur base de témoignages épigraphiques, les premiers siècles de notre ère et une ville sans doute en rapport avec la mer d'Asie Mineure, privilégiant l'origine pergaménienne. Le quatrième chapitre traite de la présence dans les Hymnes de croyances en l'au-delà qui rapporteraient ces textes à une communauté orphique. Les premières conclusions de l'A. restent, de son propre aveu, très hypothétiques : la géographie des Enfers présentée dans les Hymnes ne diffère guère de la tradition, les passages sur une vie après la mott ainsi que les demandes liées à l'au-delà sont rares et il n'y a pas de référence explicite au meurtre de Dionysos par les Titans; aussi l'A. entreprend-elle une comparaison avec les lamelles d'or, tout en reconnaissant entre ces deux types de documents un grand écart de date et une différence de fonction. Les similitudes relevées ne semblent pas toujours significatives, comme la nécessité d'être pur. Le peu de références à l'au-delà dans les Hymnes par rapport aux lamelles est expliqué par l'A. par une différence dans la diffusion, supposée plus large dans le cas des Hymnes. Le chapitre $V$ envisage la question du groupe qui eut recours à ces Hymnes : s'agit-il d'un thiase ? Comment y sont appelés ses membres ? Il ressort d'un développement serré et fondé sur une abondance de témoignages épigraphiques que les termes utilisés, comme celui de mystes, ne sont pas spécifiques à ce genre de textes et n'ont donc pas été inventés dans le cadre de ceux-ci. Bref, au terme d'un ouvrage certes intéressant mais quelque peu 
scolaire, dont un des buts, comme le dit l'A. elle-même, est de présenter le texte et de regrouper les informations qui y sont contenues, il reste encore bien des zones d'ombre autour de ces textes qui « offrent un vaste champ d'études ».

Carine Van Liefferinge (Université libre de Bruxelles

Olli Salomies (éd.), The Greek East in the Roman Context. Proceedings of a. colloquium organised by the Finnish Institute at Athens, May 21 and 22, 1999, Helsinki, 2001. 1 vol. $17,5 \times 25 \mathrm{~cm}$, II +217 p., 5 pl. (Papers and Monographs of the Finnish Institute at Athens, 7). ISBN : 951-98806-0-7.

L'analyse de l'impact du pouvoir romain sur la Méditerranée orientale - en ce compris la Grèce - a connu ces dernières années une recrudescence d'intérêt et donc une efflorescence de publications, que ce soient des monographies, des articles ou des actes de colloques. Ces Actes sont un bon reflet de l'actualité d'une telle préoccupation et réunissent un bel ensemble de spécialistes, confirmés ou à venir. Les questions religieuses ne sont toutefois pas nombreuses, ce qui justifie la brièveté de cette notice. Épinglons donc la contribution de J.-L. Ferrary (Rome et la géographie de l'bellénisme: réflexions sur. 'bellènes' et 'panbellènes' dans les inscriptions d'époque romaine) qui touche notamment à la question du Panhellénion; les deux inscriptions qui constituent le départ de cette étude ont été découvertes dans le sanctuaire de Claros et touchent à des actions

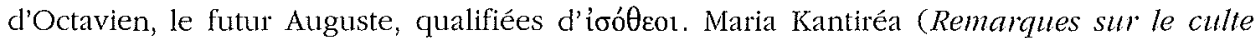
de la domus Augusta en Achaïe de la mont d'Auguste à Néron) offre une brève contribution à l'étude du culte dynastique dans la province d'Achaïe, en tentant une datation des diverses avancées en cette matière. Mika Kajava (Vesta and Atbens) pose quant à lui un très intéressant problème d'histoire religieuse : la Vesta romaine, que l'interpretatio graeca identifie comme une Hestia, avait-elle une place à Athènes, et, si oui, quel était la part de l'idéologie proprement romaine - au-delà d'un simple opportunisme politique des Athéniens - qui avait présidé à cette implantation? La démonstration est longue et parfois touffue, mais l'hypothèse d'une localisation sur l'Acropole, dans le monoptère d'Auguste et de Rome à partir de 12 av. J.-C. a de bons arguments en sa faveur. S. Follet et D. Peppas Delmousou (Les dédicaces cborégiques d'époque flavienne et antonine à Athènes), dans une contribution essentiellement épigraphique, fournissent le corpus (avec quelques photographies excellentes) remis à jour de ces inscriptions qui étaient autant de dédicaces en prose ou en vers illustrant les monuments supportant le trépied donné au vainqueur des choeurs dithyrambiques des Grandes Dionysies. P. Themelis (Roman Messene. The Gymnasium) rend compte de ses fouilles sur ce site et fait notamment référence à un certain nombre de statues divines qui s'élevaient dans le gymnase et à des inscriptions honorifiques mentionnant des fondations religieuses ou des sacrifices. D'autres contributions concernent le rapport de la 'Grèce de l'est' à la mémoire de la république romaine (C.P. Jones), les élites municipales des colonies romaines d'Achaïe (A.D. Rizakis), la famille de Théophane de Mytilène (K. Buraselis), la première province romaine de Syrie (M. Sartre, avec en annexe la liste des gouverneurs de Syrie entre 64 et 31), les inscriptions honorifiques pour les sénateurs romains en 'Grèce de l'est' (O. Salomies) et les cognomina latins dans cette région (H. Solin). Des index soigneux complètent utilement cet ensemble.

Vinciane Pirenne-Delforge (FNRS - Université de Liège)

Susan Alcock, John F. Cherry, Ja's Elsner (éds), Pausanias. Travel and Memory in Roman Greece, Oxford, University Press, 2001.1 vol. $16 \times 24 \mathrm{~cm}$, xII +379 p., 27 fig. ISBN : 0-19-512816-8.

Comme le soulignent les éditeurs dans leur préface, le présent volume trouve sa légitimation dans le regain d'intérêt dont bénéficie depuis peu Pausanias, un auteur long- 